\title{
Developing tailored and personalised diet feedback for undergraduate students delivered via the internet
}

\author{
F. Comrie and G. McNeill \\ University of Aberdeen, Aberdeen, UK
}

The purpose of the research was to design a method of providing tailored online dietary feedback from online diet assessment to promote healthy diet change in undergraduate students. Students may be at risk of developing poor diet habits because of lack of time, money, knowledge or facilities and they may not be exposed to health promotion via other routes such as workplace or community interventions. Undergraduate students are difficult to recruit to dietary surveys, since diet is not often prioritised by this group ${ }^{(1)}$. University students have excellent access to and knowledge of the internet, so this medium may be useful for measuring dietary intake and targeting individuals with diet advice. Dietary advice can be made more relevant if it is tailored and personalised. Advice is tailored if it is specifically designed to target behaviours and attitudes displayed by individuals. Personalised advice uses an individual's name or nickname ${ }^{(2)}$.

An online 121-item food-recall checklist (FoRC) was designed in Snap Survey Software (SnapSurveys Ltd, Thornbury, Bristol, UK). A database of nutrient values for foods and beverages in FoRC was developed using data from UK food composition tables ${ }^{(3)}$. Participants were recruited online to complete $4 \mathrm{~d}$ dietary assessment in FoRC as well as demographic and attitude questionnaires. FoRC provided mean individual intakes of energy (kJ), fat, non-milk extrinsic sugars, NSP (dietary fibre), fruit and vegetables, bread and breakfast cereal (g) if participants completed $\geq 3 \mathrm{~d}$ FoRC.

Individual mean food and nutrient intakes were estimated from data in FoRC and the nutrient composition values using SPSS v15.0 (SPSS Inc., Chicago, IL, USA). Mean food and nutrient intakes were uploaded into TailorBuilder v2 (OverNite Software Europe BV, Sittard, The Netherlands), a feedback generation package that matched participants' results to relevant feedback messages and created individual web pages of tailored dietary advice. Participants receiving feedback were sent an email with a link to individual web pages that detailed estimated dietary intake alongside relevant advice messages. The dietary advice messages were approved by a dietitian and were tested in a focus group.

An online diet intervention may be particularly accessible and acceptable to students who commonly use social and academic resources on the internet. Computerising data collection saved time and lowered the chance of data entry errors since data were entered only once, by participants. Median time to complete $1 \mathrm{~d}$ of FoRC was 6.1 (interquartile range 4.5-9.0) min and tailored feedback web pages could be generated for 150 participants in $<1 \mathrm{~min}$. Collecting data and tailoring and disseminating advice online was faster and more cost-effective than using paper-based methods; the workload was similar regardless of how many students took part. However, online recruitment involved less personal contact between participants and researchers and email recruitment notices may have been easier to ignore. Participants may have become fatigued by unsolicited email. Contacting $>10000$ students led to an initial recruitment of 495 students, with 285 completing stage one of the study. All data were collected over a 4-week period. Email addresses were less reliable than postal addresses for recruitment and follow-up. Unless the processes involved in diet analysis and feedback creation can be fully automated, errors can occur in database management.

Developing new methods of online dietary assessment and feedback can be time-consuming and it is difficult to check for and correct errors. Development of new methodology should allow time to thoroughly test software designs and test acceptability of information to participants. However, the use of computers and the internet means that dietary data can be collected and relevant dietary advice can be disseminated more quickly and cheaply than by paper-based methods. Internet methods may be more acceptable to the younger population who are more accustomed to computers.

1. Croll JK, Neumark-Sztainer D \& Story M (2001) J Nutr Educ 33, 193-198.

2. Kreuter MW, Farrell DW, Brennan LK \& Olevitch LR (1999) Tailoring Health Messages: Customizing Communication with Computer Technology, 1 st ed. New York: Lawrence Erlbaum Associates.

3. Food Standards Agency and Institute of Food Research (2002) McCance and Widdowson's The Composition of Foods, 6th ed. Cambridge: Royal Society of Chemistry. 